\title{
MONTE CARLO MODELING FOR AFLATOXIN B1 DISTRIBUTION IN PISTACHIO SAMPLES: A PREREQUISITE FOR SAMPLING PLAN VALIDATION
}

\author{
Nathalie Wesolek $^{1}$--- Alain-Claude Roudot ${ }^{2}$ \\ ${ }^{1,2}$ Laboratoire d'Evaluation du Risque Chimique pour le Consommateur, Université de Bretagne Occidentale, France
}

\begin{abstract}
AFB1 is the main aflatoxin found in pistachios, and as it is dangerous for health, the European Union (EU) has set a maximum level at $8 \mu \mathrm{g} / \mathrm{kg}$ in pistachios in $10 \mathrm{~kg}$ samples. Given that efficient sampling plans must be used in order to determine this contaminant level accurately, a mathematical validation of sampling plans has to be achieved with to Operating Characteristics curves. However, a prerequisite before drawing these curves is to assess the contaminant distribution within the food lot as well as to determine the variability between samples taken from the food lot. This is difficult to achieve for AFB1 in pistachios, as the contamination incidence rate is low, and concentrations differ greatly between food items. More precisely, the contaminant is heterogeneously distributed. Contrarily to current contaminant distribution assessment methods used on a regulatory basis and which are based directly on variability between samples, the method developed relied first on variability between individual pistachios, before computing the variability between samples, thus triggering more reliable results. This work assessed the distribution and incidence of aflatoxin in pistachio nuts, thanks to a Monte Carlo simulation, with pistachios split into categories, which were early split sound kernel, cracked sound kernel, and mouldy pistachios. A $30 \mathrm{~kg}$ sample size of Iranian pistachios for export to Europe was considered. Differences between good and bad lots were taken into account with lots split into 3 classes for mean contamination levels in export packages. Simulation results were in good accordance with observed contamination levels in Iran.
\end{abstract}

Keywords: AFB1, Cracked, Early split, Heterogeneous distribution, Mouldy, Mycotoxin.

\section{INTRODUCTION}

Efficient sampling plans must be used in order to determine food contaminant levels accurately. Indeed, a representative sampling plan enables to take samples from a food lot, following a given protocol, with a relatively low risk of misestimating the true mean concentration of the food lot after analysis of the food samples. A mathematical validation of sampling plans can be achieved thanks to Operating Characteristics curves (Spanjer, 2005). However, a prerequisite before drawing these curves is to assess the contaminant distribution within the food lot as well as to determine the variability between samples taken from the food lot (Whitaker et al., 1972; 2007a; 2007b) The cases for which accurate contaminant distributions are 
the most difficult to obtain are the ones with contaminants distributed heterogeneously in a food lot (Miraglia et al., 2008). In such cases, the contamination incidence rate is low, and concentrations differ greatly between food items. Such a heterogeneity problem can be observed for mycotoxins produced by moulds on granular food items like cereals, tree nuts ... Among these mycotoxins, aflatoxins, which are mainly produced by two species of Aspergillus fungi: A. flavus and A. Parasiticus (Yu et al., 1995), have genotoxic carcinogenic effects, with no threshold. They contaminate pistachios in the orchard, when Aspergillus spores, carried by insects or wind get into contact mainly with pistachios having their hull ruptured (Doster and Michailides, 1994). Indeed, the hull protects in-shell pistachios when it is not split. Finally, after industrial processing, contamination does not increase in case of proper storage condition (Miller, 1995). One type of aflatoxin: aflatoxin B1 (AFB1) is the main aflatoxin found in pistachios, as well as the most toxic. Due to its threat for human health, the European Union (EU) has set a maximum level at $8 \mu \mathrm{g} / \mathrm{kg}$ in pistachios (Regulation, 2010) in $10 \mathrm{~kg}$ samples (Regulation, 2010). Depending on harvest year, aflatoxin can cause a high rejection rate of Iranian pistachios at the point of entry into the EU. Rejections are reported through the RASFF (Rapid Alert System for Food and Feed) and cause great financial losses. A ban on Iranian pistachio imports, due to high levels of aflatoxin, occurred in September 1997. The embargo was lifted three months later following assurances that the quality of Iranian pistachio would be improved. Aflatoxin level in pistachio consignments is difficult to assess, knowing that there is a high contamination variability between individual pistachios and thus, between samples taken from the same lot. Per example, it has been estimated that only one pistachio out of 10000 to 1000000 might be highly contaminated (Schatzki and Pan, 1996.), or one out of 25000 nuts (Sommer et al., 1986). The sampling plan to be used has been validated on the basis of Whitaker's method for the determination of aflatoxin distribution and variability in tree nuts (Codex). However, this evaluation method has some limitations due to the fact that it could be misleading, as it is mainly based on experimental contamination variability data for a number of samples taken from the same lot, without considering the proportion of pistachios that are the most at risk to be contaminated. Moreover, it is derived from the analysis results of a relatively limited number of samples in comparison to the low incidence of contamination for obtaining the regression equation of sampling variance as function of lot mean concentration. For this reason, this work focuses on the development of a new method, based upon the Monte Carlo technique, for distribution and variability assessment for AFB1 in pistachios, considering prior knowledge of the contamination pattern. The Monte Carlo technique is used to estimate the effect of variability on the outputs of models which use a variety of input parameters with variability. It can be used to generate probability density functions of contamination levels obtained thanks to the combination of various distributions. The objective of this study is to model concentration distribution in $30 \mathrm{~kg}$ samples, based on specific categories of pistachios having particular visual defects and often cited in the literature as having a high probability of being contaminated. Indeed, the proportion of these categories comprised in samples, as well as their contamination probability and contamination level distribution have an impact on the variability between $30 \mathrm{~kg}$ samples taken from the same lot. Pistachio categories considered are early split sound kernel, cracked sound kernel and mouldy pistachios. Differences 
between good and bad lots are taken into account with lots split into 3 classes for mean contamination levels in samples.

\section{DATA USED AND METHODS}

\subsection{Pistachio Categories Choice}

In the orchard, when pistachios ripen, their shell splits. This is a desirable feature resulting in high value pistachios. Nevertheless at harvest stage, some pistachios, having specific characteristics, are likely to be highly contaminated by AFB1. These distinctive features are various forms of hull rupture, or kernel necrosis observed on pistachio sections or even the presence of a fungal colony. These suspicious nuts are categorized in early split sound kernel, cracked sound kernel and mouldy pistachios as described below. For the two first categories, only pistachios having sound kernels, it says non mouldy, were considered for the simulation process. It enabled to make modeling more convenient. When the hull surrounding the shell is intact at harvest, it has enabled the protection of the kernel from moulds and insects during growth stage. In case this hull is ruptured, this protection is not provided anymore, the shell is stained and the kernel can be infected by moulds. The cause of shell staining might be due to the high content of phenolic compounds in the hull (Labavitch et al., 1982). Ferguson et al. (1995) reported that shell staining generally increases with increased temperatures and increased holding times. There are two types of hull ruptures, resulting either in early split or cracked pistachios. In the first case, in the orchard, some nuts have the hull attached to the shell so that the hulls split with the shell (Doster et al., 1993). In such cases, the hull splits along the shell suture, resulting in pistachio shells stained along the shell suture. These nuts are called early splits and may be contaminated by aflatoxin (Sommer et al., 1986). However, all early split pistachios do not form at the same period. Doster and Michailides (1995) reported that even though the splitting of shell and hull for early splits tended to precede the shell splitting of normal nuts, a number of early splits formed after many normal nuts had split their shells. Even normal pistachio nuts matured unevenly over a period of several weeks during which shells split. Depending on the orchard, 15 to $48 \%$ of the early splits had formed more than 4 weeks before harvest and 10 to 30\% formed within 2 weeks before harvest. Not all early splits are infected by Aspergillus fungi, and the incidence depends on the date of splitting (Doster and Michailides, 1995). It results in even a higher variability of AFB1 contamination levels among early splits: some are contaminated while others are not. This is due to the fact that among all Aspergillus species, only A.flavus and A.parasiticus toxigenic strains have the ability to produce the toxin, and this, only under specific conditions. Furthermore, contamination levels vary between contaminated early split pistachios. The same kind of contamination variability is observed for cracked pistachios, and for the same reasons. Cracking of hulls is another type of hull rupture. This kind of hull split takes place in less than 15 days of harvest and is characterized by ragged brown edges and much wider splits than early splits and random orientation of split on hull (Pearson et al., 1994). Indeed, in cracked fruits, the location of the rupture of the hull does not correspond to the split in the shell. Early split and cracked pistachios can be either contaminated by AFB1 or not, as well as exhibit internal brown spotting or visual mold growth or not, depending on individuals. Brown spotting or visual mould growth might possibly be indicators of AFB1 contamination; however it is not always the case. Moreover, 
sometimes early split or cracked pistachios having apparently sound kernels are contaminated. And on the contrary, pistachios having suspect kernels and/or split hulls might not be contaminated. For these reasons, as mentioned earlier, for modeling purposes, we only considered early split pistachios having sound kernels and cracked pistachios having sound kernels mentioned as early split sound kernel category and cracked sound kernel category, respectively. Steiner et al. (1992) observed that some brown or brown spotted pistachio kernel sections in pistachio nuts cut lengthwise with a knife, were highly contaminated with aflatoxin. These spots can be either typical kernel necrosis symptoms or indication of fungal growth. As regards kernel necrosis symptoms, they are caused by large bugs' feeding named hemipteras. According to Michailides et al. (1995), kernel necrosis symptoms differ from stigmatomycosis, which is characterized by the wet, smelly, rancid, slimy appearance of the kernel. Typical kernel necrosis symptoms caused by hemipteras are dry, punchy, areas in the kernel, spongy at times, usually appearing in the area close to the stem and or along the split line of the shell (Danisti et al., 2001). The most critical period for potential kernel damage occurs in midseason, when fruit load is set, shell hardening is not complete and large bugs' mouthparts can easily penetrate hardening shells. The damaged nut remains in the cluster and the feeding punctures result either to epicarp lesion staining the outer shell or to necrotic spots on the kernels contaminated by fungi (Daane et al., 2005a). The subsequent feeding by large bugs presents potential "hidden" damage, because kernel necrosis may occur without the external sign (hull lesion) of insect feeding. Late-season feeding can also lead to kernel necrosis when the insect's mouthparts penetrate the shell near the "Achilles heel" of the pistachio, the region near the peduncle (nut's stem) (Michailides et al., 1988). The contamination pathway of pistachios by A. flavus or parasiticus following bugs' feeding is probably the same as by another fungus, Botryosphaeria dothidea $(\mathrm{Bd})$, a panicle blight of pistachios. Daane et al. (2005b) reported that for Bd, the most probable contribution of bugs is providing germination sites for the fungus: by puncturing the epicarp with their mouthparts, the bugs allow spores that are already present to germinate. Hadavi (2005) concluded that in-orchard contaminations probably obey the same rules as inoculation tests, which are, i.e. the fungus gains entry from any kind of splits in hull and grow between hull and shell space until reaching to nut stem and after penetrating via numerous microscopic passages here, the fungus reaches the kernel in the placental region. The fungus possibly follows the least physical restriction with adequate moisture path, which directs it to intra-cotyledonary space, the observed brown colour or spots here, probably may act as an indicator of physical growth and/or restriction pattern of fungus (Hadavi, 2005). In our work, we put pistachios having brown or brown spotted pistachio kernel sections under the mouldy category.

\subsection{Global Steps of the Method}

The modeling method developed in this work used the Monte Carlo technique, and comprised many steps as described hereafter. The cumulative distribution functions of contamination levels in individual pistachios corresponding to each category (early split sound kernel; cracked sound kernel; mouldy) were shown to be linear with a positive slope in graphical representations with lognormal distribution probability scales. The lognormal distributions were then used to calculate $30 \mathrm{~kg}$ samples contamination levels for a given number of contaminated 
individuals per sample. Differences between good and bad lots were taken into account with lots split into 3 quality lot classes. The following calculations were achieved for each quality lot class separately, knowing that the various pistachio category incidences vary between quality lot classes. First, the probability of having a specific number of contaminated individuals within each specific category part of a $30 \mathrm{~kg}$ sample was determined thanks to a binomial distribution, taking into account the contamination incidence. Then, a probability distribution was used to combine probabilities and contamination level distributions formerly calculated. As a result, $30 \mathrm{~kg}$ samples contamination distributions, which take all the possible number of contaminated individuals into account, were obtained. Finally, these contamination distributions for each category were added up, thereby giving the distribution of $30 \mathrm{~kg}$ samples contamination levels for each quality lot class. Following this, the three obtained distributions (one for each quality lot class) were combined thanks to binomial distributions: the two lowest quality lots classes were first combined, and then the result was combined to the highest quality lot class.

\subsection{Contamination Incidence and Levels}

Contamination incidence and levels in individual pistachios were calculated from results reported in a publication from Hadavi (2005), in which all aflatoxin contaminations were due to $A$. flavus, thus resulting in AFB1 contamination. In the study we refer to, pistachios were collected from six commercial orchards in Iran and aggregated into samples, based upon hull appearance. It is reported that, by this sampling procedure, the heterogeneous distribution of contaminated nuts was well covered, as practiced earlier in literature (Hadavi, 2005). Sound, early split, cracked (growth split) pistachio samples for each orchard were separated according to fluorescence or non-fluorescence, as well as kernel aspect. Indeed, the bright greenish yellow colour under an ultraviolet light, referred to as the 'BGY' fluorescence (BGYF) is partially linked to aflatoxin contamination (Hadavi, 2005). The published data taken into account for our simulation, but with slight modification, were sample contamination levels for early split and cracked pistachio samples having sound kernels, as well as pistachios having brown spotted or mouldy kernels, or with the presence of fungal colony. More precisely, in our work, pistachios were categorized according to visual defects into three categories, namely early split sound kernel, cracked sound kernel and mouldy pistachios. As the sample size was small enough (from $1.1 \mathrm{~g}$ to $168 \mathrm{~g}$ of kernels), we considered that a maximum of one kernel per sample was contaminated, taking into account a kernel weight of $0.52 \mathrm{~g}$. Indeed, aflatoxin incidence is a rare event in pistachios, even in mouldy kernels, due to the fact that Aspergillus is not the sole mould capable of infesting the nut. In addition, when toxigenic Aspergillus is present, toxin is only synthetized in specific conditions. Then, the calculation of the incidence of contaminated pistachios per category and individual contamination levels was achieved. Individual contamination level distributions were fitted to theoretical distributions for each pistachio category. We made the hypothesis, for the contamination incidence calculation, that a maximum of one kernel per sample was contaminated. In order to further validate this assumption, we compared the contamination incidence of early split pistachios (having sound or brown spotted kernels) calculated from Hadavi (2005) data to the incidence level calculated from other publications. This latest calculation was made by multiplying the incidence level of $2 \%$ of pistachios with $A$. flavus among early split pistachios 
reported by Bonjar (2004) for the 2003 crop year by the proportion of toxigenic fungi among $A$. flavus isolates from pistachios. Indeed, Rahimi et al. (2008) reported that among 150 isolates of $A$. flavus tested for aflatoxin production, 46 were identified as aflatoxin producing isolates. Calculated individual contamination levels were compared to data from a publication from Mahoney and Rodriguez (1996). In their study, they used mature pistachios with undamaged and nonadhering hulls, collected from an orchard for in vitro inoculations. Their protocol is in the following paragraph:

The hulls were aseptically sliced along the shell suture to simulate early-split pistachios on 100 nuts, and pistachios with unopened shells were discarded. The hulls and shells were aseptically removed from other 200 nuts. One-half of all kernels were injured by using a scribe to make a puncture wound approximately $1 \mathrm{~mm}$ wide by $2 \mathrm{~mm}$ deep in each kernel. Pistachios were placed in petri dishes (five nuts per plate). Each nut was inoculated on the kernel (or the wound in the kernel) with approximately 200 spores of $A$. flavus aflatoxigenic strain. The petri plates were incubated for 10 days at $25^{\circ} \mathrm{C}$.

From these published data, we transformed aflatoxin levels in individual pistachios given in $\mu \mathrm{g} / \mathrm{kernel}$ in order to obtain concentration levels in $\mu \mathrm{g} / \mathrm{kg}$. Then, data for contaminated hull sliced pistachios (intact or wounded kernels) were fitted to a theoretical distribution. Mean and standard deviation obtained were compared to our simulation results calculated for the early split sound kernel category. We considered that the theoretical distribution of contaminated hulled pistachios would be the same as for hull sliced pistachios. We simulated the distribution for hulled intact kernel pistachios and hulled wounded kernel pistachios, using@Risk (version 6, Palissade Corp.). Finally, we combined the two distributions, using a binomial distribution. Mean and standard deviation of the resulting distribution were compared to our simulation results for mouldy category pistachios. Another validation was made by comparing the incidence of highly toxigenic $A$. flavus strains in early split pistachios (resulting in a concentration level $>200 \mu \mathrm{g} / \mathrm{kg}$ in a pistachio) calculated from the contamination incidence calculation under study for Iranian pistachios to the same kind of data from USA. Indeed, Pearson (1996) reported for the USA, from a publication from Sommer et al. (1986), that the incidence of aflatoxin contamination greater than $200 \mu \mathrm{g} / \mathrm{kg}$ in individual early split nuts was approximately 1 in 270 . Knowing the incidence of early split pistachios contaminated by $A$. flavus (either toxigenic or not) for both countries: $2 \%$ in Iran (Bonjar, 2004), 0.7\% in USA (Doster and Michailides, 1994), we deduced the proportion of early split pistachios with $A$. flavus that have a highly toxigenic strain of the fungus. We assume that the incidence of a highly toxigenic $A$. flavus in individual early split pistachios contaminated by $A$. flavus is supposed to be the same in both countries (Iran and USA). If so, it validates the strategy used in this work for contamination incidence and levels obtainment.

\subsection{Monte Carlo Technique Using @Risk}

The @Risk software (version 6, Palissade Corp.) is a Monte Carlo software for risk analysis and risk assessment using Monte Carlo random simulations. It is used to determine how often specified events may occur thanks to the generation of Probability Density Functions (PDFs). This probability risk analysis approach is opposed to the deterministic risk analysis calculation. Indeed, when achieving a deterministic risk evaluation, values are assigned for discrete scenarios to see 
what the outcome might be in each. On the contrary, in a probability risk evaluation, values are given to all the possible scenarios, taking into account their probability of occurrence. It is used to estimate the effect of variability on the outputs of models which use a variety of input parameters. Monte Carlo simulation is the replication of a procedure with input data drawn randomly from a parametric distribution. However, Monte Carlo simulation can be used with two different sampling types under @ Risk: the Monte Carlo (MC) and the Latin Hypercube (LH) sampling types. The LH sampling is a particular MC sampling: more precisely it is a constrained MC (i.e. random) sampling scheme, involving a stratification of the input distribution. The constraint refers to the way each variable is sampled: the statistical cumulative distribution is split in n equally probable intervals on the cumulative probability scale, and then a random value is selected within each interval. The number of $\mathrm{n}$ equally probable interval is equal to the number of iterations of the Monte Carlo simulation. A simulation with 500 iterations, per example, would split the probability into 500 segments, each representing $0.2 \%$ of the total distribution. For the first segment, a number would be chosen between $0.0 \%$ and $0.2 \%$. For the second segment, a number would be chosen between $0.2 \%$ and $0.4 \%$. This number would be used to calculate the actual variable value based upon its distribution. Once each variable has been sampled using this method, a random grouping of variables is selected for each Monte Carlo calculation. Independent uniform selection is done on each of the variable's generated values. Each value must only be used once. LH sampling would create a more representative sampling of the distribution, ensuring that tail samples of the input distributions are drawn (e.g. for 1000 iterations, exactly one value above the P99.9 would be drawn, whereas for MC sampling either none, one or several samples may be drawn). So, LH sampling can be used to force the sampling of tails of distributions, along with a great number of iterations. This is why, in this study, thanks to the @Risk 5.7 software (Palisade Corp.), PDFs were modeled, using Monte Carlo simulations under the LH sampling type. The number of iterations was 100,000 to attain a very high stability level.

\subsection{Modeling of Pistachio Categories and Contamination}

Simulated lots were split into three different quality levels for the mean contamination level, numbered by ascending contamination level: $1^{\text {st }}$ class, $2^{\text {nd }}$ class, $3^{\text {rd }}$ class. We considered a constant incidence level of pistachio categories within lots of the same quality levels. The incidence of pistachio categories used for pistachios was deduced from a document from the UNECE (United Nations Economic Commission for Europe), defining the maximum tolerances for « extra pistachios » (UNECE, 2001): 2\% of early split pistachios and 1\% of cracked pistachios. So, for $2^{\text {nd }}$ and $3^{\text {rd }}$ class lots, the incidence levels of early split sound kernel and cracked sound kernel pistachios were set at $2 \%$ and $1 \%$ respectively. However, for $1^{\text {st }}$ class lots, these categories incidences were divided by 2 . As regards the incidence of the mouldy pistachio category, we kept it as an adjustment variable. The final calculation of contamination incidence was achieved thanks to the Bayes' theorem in Equation (1):

$$
P(\text { contaminated } \cap \text { category })=P(\text { contaminated / category }) \times P(\text { category })
$$

with $P$ (contaminated), the probability that the individual is contaminated and $P$ (category), the probability that the individual is in a given category. 
Calculations were achieved for each pistachio category separately, at first. The contamination distribution for $30 \mathrm{~kg}$ samples for a given number of contaminated individuals per sample is given in Equation (2):

$$
C s=\frac{C i}{N i} \times N i c
$$

With $C s$, $30 \mathrm{~kg}$ samples concentration distribution; $C i$, individual pistachios concentration distribution; $\mathrm{N} i$, the number of individual inshell pistachios in a $30 \mathrm{~kg}$ sample; Nic, the number of contaminated individual inshell pistachios in a $30 \mathrm{~kg}$ sample. The probability of a given number of contaminated individuals $(0,1,2,3$, etc.) was calculated thanks to a binomial distribution, knowing the final calculation result of the contamination incidence. These probabilities were then grouped together. A conditional function was used to match these probabilities with concentration levels distributions in $30 \mathrm{~kg}$ samples. The result was a PDF for the contamination level in $30 \mathrm{~kg}$ samples, considering only the contamination stemming from one pistachio category. In a second step, PDFs obtained for each pistachio category were added up in order to obtain the PDFs of final concentration in $30 \mathrm{~kg}$ samples for each of the 3 classes. The three PDFs obtained for the quality lot classes were combined thanks to binomial distributions. More precisely, the two lowest quality lots classes ( $2^{\text {nd }}$ and $3^{\text {rd }}$ class) were first combined, and then the result was combined to the highest quality lot class ( $1^{\text {st }}$ class). However, various probability values to use for the binomial distributions were tested. The values chosen were the ones that gave a final PDF for contamination levels in $30 \mathrm{~kg}$ samples as close as possible to the observed values in Iran as further detailed in 2.6.

\subsection{Simulation Adjustment}

Our simulation final parameters were the binomial probabilities to put the three quality lot classes together. These parameters were chosen in such a way that the resulting output final PDF for contamination levels in $30 \mathrm{~kg}$ samples was adjusted to a contamination distribution observed in Iran, reported by Cheraghali et al. (2007). Their distribution was obtained for pistachio nuts produced in Iran during March 2002-February 2003 and analyzed for AFB1. Lots sampled were pistachio nut consignments, intended for export to EU, that are usually about 25 tons. Their sampling plan was the following: taking one $30 \mathrm{~kg}$ sample (100 incremental samples, each $300 \mathrm{~g}$ ), mixing and division to three $10-\mathrm{kg}$ sub-samples. They collected 3,356 pistachio nut samples of 30 $\mathrm{kg}$, and after dividing samples to $10 \mathrm{~kg}$ sub-samples, performed 10,068 AFB1 analyses. However, the mean of the contamination level in sub-samples was used in their study, which is the same as if they had analyzed $30 \mathrm{~kg}$ samples directly.

\section{RESULTS AND INTERPRETATION}

\subsection{Contamination Incidence and Levels}

Contamination levels in individual pistachios were calculated from contamination levels in small samples reported by Hadavi (2005) for contaminated samples only. Results are given in Table 1 and were obtained by multiplying contamination levels in the samples by the corresponding number of pistachios in the samples (deduced from total kernel weight) for each pistachio category. Furthermore, the calculation of the incidence of contaminated pistachios per 
category was achieved: $P$ (contaminated / category)), it says the probability that an individual pistachio was contaminated, knowing that it was in a specific category. Considering that a maximum of one kernel per small sample was contaminated, in agreement with section 2.3, the result for the probability of contamination within each category, was equal to $7.53425^{*} 10^{-3}$, $6.709265^{*} 10^{-2}$, and $6.14035^{*} 10^{-3}$, for early split sound kernel, mouldy, and growth split sound kernel categories, respectively. Goodness of fit tests were achieved for calculated individual contamination levels (reported in Table 1) for each pistachio category, using@Risk. Test statistics for the Kolmogorov-Smirnov (K-S) test for lognormal distribution, triggered by the software were compared to critical values for this test at a $5 \%$ risk level. As each test statistic value is lower than its corresponding critical values, a lognormal distribution cannot be rejected as the true parent distribution at a $5 \%$ risk level. For a visual assessment, cumulative distribution functions (CDF) for contamination levels, were plotted using the R software in Web Figure 1, 2, 3 for early split sound kernel, cracked sound kernel and mouldy pistachio categories, respectively, in graphs with a standard normal probability scale and a $\log$ axis for the data. Least square regression lines were obtained for each graph and show a low dispersion level. As, with such a graphical representation, any given lognormal CDF plots as a straight line with positive slope, we further confirm that the distributions under study fit lognormal distributions. Mean contamination level was at $5542 \mu \mathrm{g} / \mathrm{kg} \pm 10525 \mathrm{SD} ; 2527 \mu \mathrm{g} / \mathrm{kg} \pm 3412 \mathrm{SD}$; and $287646 \mu \mathrm{g} / \mathrm{kg}$ \pm 706790 for contaminated early split sound kernel, cracked sound kernel, and mouldy pistachios, respectively. So, mean individual contamination level is higher in early split sound kernel than in cracked sound kernel pistachio category, the highest mean contamination being in mouldy pistachio category.

Indeed, Doster and Michailides (1994) reported that most early split nuts had the hull split before any pistachio fruits had cracked hull, which may explain why cracked fruits have less kernel mould than early splits. The fact that early split and cracked pistachio categories (having apparently sound kernels) are contaminated can be explained by the fact that the mould is not present anymore, but has synthetized aflatoxins while it has been growing. Considering a maximum of one contaminated kernel per sample in data from Hadavi's publication, we calculated the contamination incidence of early split pistachios (having sound or brown spotted kernels), as being 23 in 1672 , leading to a value of $1.37 \%$. This value was compared to another calculation result of the same parameter derived from Bonjar (2004) and Rahimi et al. (2008): $0.30 \times 2 \%=$ $0.60 \%$. As $1.37 \%$ is in the same order of magnitude as $0.60 \%$, we can deduce that contamination incidence values, as calculated for our simulation must be very close to what could have been observed if individual pistachios had been analyzed for aflatoxin. Indeed, aflatoxin analyses are very costly and thus seldom achieved on individual pistachios. And even though individual pistachios were analyzed in few literature data, these analyses were only achieved on a limited number of individuals. For this reason, we consider contamination data from small samples for our simulation. As the calculated incidence from small samples reported above fit well with calculated incidence derived from fungal incidence and toxicity expectations, we can deduce that our contamination incidence calculation thanks to small samples data is validated. Calculated individual contamination levels for our simulation were compared to in-vitro data from a publication from Mahoney and Rodriguez (1996). Their contamination data for hull sliced 
pistachios, having the same aspect as early splits, were fitted to a lognormal distribution, for contaminated individuals only, using @Risk. The test statistic value for the Kolmogorov-Smirnov (K-S) test was equal to 0.0998. It is lower than the critical value (0.1831) for this test at a $5 \%$ risk level. So, a lognormal distribution cannot be rejected as the true parent distribution at a $5 \%$ risk level. Mean level obtained was at $4565 \mu \mathrm{g} / \mathrm{kg}$, with a standard deviation (SD) equal to 6092 . These values are very close to the corresponding parameters obtained with our simulation for early split sound kernel category. Furthermore, their data for contaminated hulled pistachios were tested too. We considered that both hulled intact kernel and hulled wounded kernel contamination levels would fit a lognormal distribution. So, we simulated the distributions for hulled intact kernel pistachios (mean $=46519 \mu \mathrm{g} / \mathrm{kg} ; \mathrm{SD}=164942)$ and hulled wounded kernel pistachios $($ mean $=557692 \mu \mathrm{g} / \mathrm{kg} ; \mathrm{SD}=430153)$, using @ Risk. Then, we combined the two distributions, using a binomial distribution, with a probability set at 0.5. The resulting distribution $($ mean $=299502 \mu \mathrm{g} / \mathrm{kg} ; \mathrm{SD}=406013)$ is very similar to the one obtained with our simulation for mouldy pistachio category. Thanks to all these results, we can validate the process used for our simulation, for contamination level distribution obtainment. From Hadavi's data, the incidence of aflatoxin contamination greater than $200 \mu \mathrm{g} / \mathrm{kg}$ in individual early split nuts was 15 in 1672 , corresponding to a result of 1 in 111 . Knowing that the incidence of early split pistachios contaminated by $A$. flavus (either toxigenic or not) in Iran was $2 \%$ (Bonjar, 2004), we calculated that $45 \%$ of early split pistachios contaminated by $A$. flavus were contaminated by a highly aflatoxigenic strain. The same calculation was made for pistachios from USA, as described in paragraph 2.3., and the corresponding final result was $53 \%$ for the proportion of early split pistachios contaminated by $A$. flavus that are contaminated by a highly aflatoxigenic strain. As both figures for Iran and USA are rather similar, we can validate the strategy used in this work for contamination incidence and levels obtainment.

\subsection{Contamination Levels Distribution in Samples}

As reported in paragraph 2.5, for $1^{\text {st }}$ quality lot class, the incidence levels of early split sound kernel and cracked sound kernel pistachios were set at $1 \%$ and $0.5 \%$, respectively. For $2^{\text {nd }}$ and $3^{\text {rd }}$ class lots, the incidence levels of early split sound kernel and cracked sound kernel pistachios were set at $2 \%$ and $1 \%$, respectively. In order to cover the whole range of $\mathrm{AFB} 1$ levels observed in $30 \mathrm{~kg}$ samples in the publication used for simulation adjustment (reported in paragraph 2.6.), incidence levels of mouldy pistachios were set at $0 \%, 0.01 \%$, and $0.25 \%$ for $1^{\text {st }}, 2^{\text {nd }}$ and $3^{\text {rd }}$ quality lot classes. The corresponding calculation result of final contamination incidence, achieved with Equation (1) and probability of contamination data, is shown in Table 2. Then, calculations were achieved as described in paragraph 2.5. Thanks to these data, PDFs were simulated using@Risk, for each quality lot class, as described below. The contamination PDF in $30 \mathrm{~kg}$ samples from $1^{\text {st }}$, $2^{\text {nd }}$ and $3^{\text {rd }}$ quality lot class are given in Figure 1,2 and 3 , respectively. In order to enable the comparison with Cheraghali's data, the proportion of samples in various intervals is given in Table 3. When comparing the 3 quality lot classes' distributions given in Table 3, it is noticeable that, for the 3 highest contamination intervals, $3^{\text {rd }}$ quality lot class samples might have the greatest impact, if all 3 quality lot classes were put together. Indeed, the 3 quality lot classes had to be mixed up to fit simulation data to observed results (Cheraghali's data), but prior to this, it 
was important to ascertain that the whole range of observed concentrations is well covered. In the same way, for the lowest contamination interval, both $1^{\text {st }}$ and $2^{\text {nd }}$ quality lot classes have probably the highest impact, whereas for $2.01-5 \mu \mathrm{g} / \mathrm{kg}$ and $5.01-20 \mu \mathrm{g} / \mathrm{kg}$ intervals, both $2^{\text {nd }}$ and $3^{\text {rd }}$ quality lots have the biggest weight. These observations are further examined in Web Figure 4.

From this figure, we observed that the range of observed concentrations (in Cheraghali's data) is well covered by the 3 simulated quality lot classes. However, simulation adjustment reported in paragraph 2.6 had to be achieved, to combine the 3 quality lot classes together.

\subsection{Final Simulation Adjustment and Final Contamination Levels in Samples}

A binomial distribution was used to combine the $2^{\text {nd }}$ and $3^{\text {rd }}$ lot classes together with a probability at 0.6 for the $2^{\text {nd }}$ lot class. This combination was then mixed with the $1^{\text {st }}$ lot class, this latter class having a probability set at 0.8. Simulated and observed (from Cheraghali’s data) PDFs for final concentrations in $30 \mathrm{~kg}$ samples in export packages are compared in Web Figure 5. For $30 \mathrm{~kg}$ samples above $500 \mu \mathrm{g} / \mathrm{kg}$, the observed percentage was $0.07 \%$ and the simulated percentage at $0.1 \%$. The simulation was validated, as the probabilities were very similar in both simulated and observed distributions. Moreover, obtained mean and SD for the simulation were equal to 5.2 and $45.9 \mu \mathrm{g} / \mathrm{kg}$, respectively, whereas the corresponding values from observed PDF were 5.9 and $41.7 \mu \mathrm{g} / \mathrm{kg}$, respectively.

\section{DISCUSSION}

Due to the low contamination incidence in individual pistachios, AFB1 concentration levels differ greatly between $30 \mathrm{~kg}$ samples. Our strategy in this modeling work is to split lots between quality classes as regards concentration levels. These categories are chosen as they are highly susceptible of being contaminated by AFB1. We postulate that using analytical data for small samples of each pistachio category and then deriving the concentration levels in $30 \mathrm{~kg}$ samples, as achieved in our study, is more reliable than using directly $10 \mathrm{~kg}$ or $30 \mathrm{~kg}$ samples analytical data, as usually performed in Whitaker's method. As the contaminant is heterogeneously distributed, it appears that small samples of pistachios having the same defects must have a more homogeneous contamination distribution than big samples of any pistachio type (not split into categories). Furthermore our simulation method uses the Monte Carlo technique, thereby enabling random simulations, as well as the combination of various distributions. When our results are compared to contamination levels observed in Iran, both distributions are very similar. A sensitivity analysis must be performed with@Risk, to show the sensitivity of the output contamination distribution to its input variables. The goal is to identify and rank important sources of variability. The only parameters that are set in the simulation in order to fit the final observed distribution and that are used as adjustment variables are the binomial probability levels. Indeed, these binomial probabilities are used to combine the 3 quality lot classes' distributions together. All the other parameters, used in our model, are obtained or derived from analytical data or regulatory levels. For this reason, it is necessary to assess whether the binomial probability levels are important sources of variability in the model or not. Sensitivity analysis results show that the regression coefficient between the output distribution and these two input variables have a global value of $13 \%$. So, the two input variables are low sensitivity variables, meaning that even large 
changes to the distribution of these variables may have a minimal impact on the final result. In other words, the binomial probability levels used do not have a high influence on the output contamination distribution. Then, a sensitivity analysis targeted on the variation of these two input variables was achieved. In order to better comprehend and quantify the impact of the binomial probability levels chosen, we set them at different values and observed the effect on the output contamination distribution. The binomial probability levels for each variable were set at their initial value \pm 0.1 , their initial value being the value used for final simulation adjustment (paragraph 3.3). So, for the first input variable under study, the probability value levels tested for the $2^{\text {nd }}$ lot class were $0.5,0.6$ or 0.7 . As regards the second input variable, the probability value for the $1^{\text {st }}$ lot class was set at 0.7 , 0.8 or 0.9 . All the output distributions for final contamination levels from our model, resulting from each of the possible combinations of the two variables under study were simulated using@Risk. The percentage of samples for various contamination levels was obtained. Then, the minimum and maximum percentage values for each contamination interval level were compared to the percentage values for the model previously validated (paragraph 3.3). Results are plotted in Web Figure 6, with minimum and maximum values represented as lines on the bars to show some aspects of variability on the developed model. This sensitivity analysis confirms that when the two binomial probability levels vary, the impact on the output distribution is very low. Thus, the model developed in our study is robust. The impact of this work might be interesting from a prevention point of view, as shown hereafter. The model is based on contamination levels in specific pistachio categories, and the proportion of these categories within a lot is function of the mean contamination level of the lot. So, estimating the proportion of pistachio categories within each lot gives an estimation of lot mean contamination level.

This simulation uses AFB1 contamination levels from early split pistachios and cracked pistachios having sound kernels, as well as from pistachios having brown-spotted kernel sections due to kernel necrosis or mould infection. All these pistachios are dubious nuts, meaning that they are highly susceptible of being contaminated. Observed contamination levels distribution in samples taken from lots from Iran, come from the presence of these nuts. Indeed, contamination levels per contaminated pistachios within a sample are cumulated and must be divided by the total sample weight to obtain sample concentration level.

\section{CONCLUSION}

Contamination incidence and levels in individual pistachios used in our simulation were validated thanks to calculations made from other literature data. They were then combined together to simulate contamination PDFs in $30 \mathrm{~kg}$ samples of three different classes of pistachio lots. The final result was the contamination distribution in $30 \mathrm{~kg}$ samples, adjusted thanks to contamination data from Iran. So, as the simulation truly reflects observed contamination levels distribution in samples from Iran, it validates our understanding of the points that bring contamination variability. By this way, this work is going to enable to predict sample distribution better and thus to validate sampling plans more accurately. 


\section{ACKNOWLEDGEMENTS}

This project was partly financed by the BASELINE European research project, which is part of the $7^{\text {th }}$ Framework Programme (grant agreement 222738). This project is titled "Selection and improving of fit-for-purpose sampling procedures for specific foods and risks" and spans from 2009 to 2013.

\section{REFERENCES}

Bonjar, G.H.S., 2004. Incidence of aflatoxin producing fungi in early split pistachio nuts of Kerman, Iran. Journal of Biological Sciences, 4(2): 199-202.

Cheraghali, A.M., H. Yazdanpanah, N. Doraki, G. Abouhossain, M. Hassibi, S. Ali-Abadi, M. Aliakbarpoor, M. Amirahmadi, A. Askarian, N. Fallah, T. Hashemi, M. Jalali, N. Kalantari, E. Khodadadi, B. Maddah, R. Mohit, M. Mohseny, Z. Phaghihy, A. Rahmani, L. Setoodeh, E. Soleimany and F. Zamanian, 2007. Incidence of aflatoxins in Iran pistachio nuts. Food and Chemical Toxicology, 45(5): 812-816.

Codex, Development of aflatoxin sampling plans for Almonds, Hazelnuts, Pistachio, and Brazil Nuts. Technical information provided by the U.S. delegation to members of the electronic working group to help recommend aflatoxin sampling plan designs for treenuts to the full committee. Available from http://www.bae.ncsu.edu/usda/www/ResearchActDocs/CCCF1.pdf [Accessed 10/01/13].

Daane, K.M., J.G. Millar, R.E. Rice, P.G. Da Silva, W.J. Bentley, R.H. Beede and G. Weinberger, 2005b. Stink bugs and leafooted bugs. In pistachio production manual, 4th Edn., Ferguson L, Beede RH, Freeman MW, Haviland DR, Holtz BA, Kallsen CE, Coviello JA (ed). Pest, disease and physiological disorders management, pp: 186-196. Available at: fruitsandnuts.ucdavis.edu/files/73703.pdf.

Daane, K.M., G.Y. Yokota, R. Krugner, S.A. Steffan, P.G. Da Silva, R.H. Beede, W.J. Bentley and G.B. Weinberger, 2005a. Large bugs damage pistachio nuts most severely during midseason. California Agriculture, 59(2): 95-102.

Danisti, L., A. Eskalen, S. Karadag and M. Kusek, 2001. Fungal diseases in pistachio trees in EastMediterranean and Southeast Anatolian regions. In: Ak B.E. (ed.). XI GREMPA Seminar on Pistachios and Almonds. Zaragoza : CIHEAM. pp: 261-264 (Cahiers Options Méditerranéennes; No 56).

Doster, M.A. and T.J. Michailides, 1994. Aspergillus molds and aflatoxins in pistachio nuts in California. Postharvest Pathology and Mycotoxins, 84(6): 583-590.

Doster, M.A. and T.J. Michailides, 1995. The relationship between date of hull splitting and decay of pistachio nuts by aspergillus species. Plant Disease, 79(8): 766-769.

Doster, M.A., T.J. Michailides and D.A. Goldhamer, 1993. Influence of cultural practices on occurrence of early split pistachio nuts. California pistachio industry, annual report crop Year 1992-1993. California Pistachio Commission, Fresno. pp: 82-84.

Ferguson, L., A. Kader and J. Thompson, 1995. Harvesting, transporting, processing and grading. In pistachio production; Ferguson, L. Ed.; Center for fruit and nut crop research and information, Pomology Department, University of California: Davis, CA. pp: 110-114. 


\section{Journal of Food Technology Research, 2014, 1(1): 1-20}

Hadavi, E., 2005. Several physical properties of aflatoxin-contaminated pistachio nuts: Application of BGY fluorescence for separation of aflatoxin-contaminated nuts. Food Additives and Contaminants, 22(11): 1144-1153.

Labavitch, J.M., C.M. Heintz, H.L. Rae and A.A. Kader, 1982. Physiological and compositional changes associated with maturation of 'Kerman' pistachio nuts. Journal of the American Society for Horticultural Science, 107(4): 688-692.

Mahoney, N.E. and S.B. Rodriguez, 1996. Aflatoxin variability in pistachios. Applied and Environmental Microbiology, 62(4): 1197-1202.

Michailides, T.J., D.P. Morgan and M.A. Doster, 1995. Diseases of pistachios in California and their significance, In N. Kaska, A. B. Kuden, L. Ferguson, and T. Michailides (eds.), 1st International Symposium on Pistachio. ISHS Acta Horticulturae 419, Adana, Turkey. pp: 337-343

Michailides, T.J., J.M. Ogawa and R.E. Rice, 1988. Sites of epicarp lesions and kernel necrosis in relationship to symptoms and phenology of pistachio fruit. Journal of Economic Entomology, 81(8): 1152-1154.

Miller, J.D., 1995. Fungi and mycotoxins in grain: Implications for stored product research. Proceedings of the 6th International Working Conference on Stored-product Protection, 2: 971-977.

Miraglia, M., B. De Santis, E. Pannunzi, F. Debegnach and C. Brera, 2008. Mycotoxin concentration data quality: The role of sampling. In Mycotoxins: Detection Methods, Management, Public Health, and Agricultural Trade. Eds. Leslie, J.F., Bandyopadhyay, R., Visconti, A. pp: 185-193.

Pearson, T., 1996. Machine vision system for automated detection of stained pistachio nuts. LebensmittelWissenschaft undTechnologie, 29(3): 203-209.

Pearson, T.C., D.C. Slaughter and H.E. Studer, 1994. Physical properties of pistachio nuts. Transactions of the ASAE, 37(3): 913-918.

Rahimi, P., B. Sharifnabi and M. Bahar, 2008. Detection of aflatoxin in aspergillus species isolated from pistachio in Iran. Journal of Phytopathology, 156(1): 15-20.

Regulation, 2010. (EU) No 165/2010 of 26 February 2010 amending Regulation (EC) No 1881/2006 setting maximum levels for certain contaminants in foodstuffs as regards aflatoxins. Official Journal of the European Union L, 50(27): 8-12.

Regulation, 2010. (EU) No 178/2010 of 2 March 2010 amending Regulation (EC) No 401/2006 as regards groundnuts (peanuts), other oilseeds, tree nuts, apricot kernels, liquorice and vegetable oil. Official Journal of the European Union L, 52(3): 32-43.

Schatzki, T.F. and J.L. Pan, 1996. Distribution of aflatoxin in pistachios. 3. Distribution in pistachio process streams. Journal of Agriculture and Food Chemistry, 44(4): 1076-1084.

Sommer, N.F., J.R. Buchanan and R.J. Fortlage, 1986. Relation of early splitting and tattering of pistachio nuts to aflatoxin in orchards. Phytopathology, 76(7): 692-694.

Spanjer, M.C., 2005. Sampling and sample preparation: An underestimated issue ? In rapid methods for biological and chemical contaminants in food and feed. Eds. Van Amerongen, A., Parug, D., Lauwaars, M. pp: 39-63.

Steiner, W.E., K. Brunschweiler, E. Leimbacher and R. Schneider, 1992. Aflatoxins and fluorescence in Brazil nuts and pistachio nuts. Journal of Agricultural and Food Chemistry, 40(12): 2453-2457.

UNECE, 2001. Revision of UNECE standards inshell pistachio nuts, transmitted by Turkey, TRADE/WP.7/GE.2/2001/6, DF-09, pp: 1-10. 
Whitaker, T.B., J.W. Dickens, R.J. Monroe and E.H. Wiser, 1972. Comparison of the observed distribution of aflatoxin in shelled peanuts to the negative binomial distribution. Journal of the American Oil Chemists' Society, 49(10): 590-593.

Whitaker, T.B., J.J. Saltsman, G.M. Ware and A.B. Slate, 2007a. Evaluating the performance of sampling plans to detect hypogycin a in ackee fruit shipments imported to the United States. Journal of AOAC International, 90(4): 1060-1072.

Whitaker, T.B., A.B. Slate, J.M. Hurley and F.G. Giesbrecht, 2007b. Sampling almonds for aflatoxin, part II: Estimating risks associated with various sampling plan designs. Journal of AOAC International, 90(3): 778-785.

Yu, J., P.K. Chang, J.W. Cary, M. Wright, D. Bhatnagar, T.E. Cleveland, G.A. Payne and J.E. Linz, 1995. Comparative mapping of aflatoxin pathway gene clusters in aspergillus parasiticus and aspergillus flavus. Applied and Environmental Microbiology, 61(6): 2365-2371.

Table-1. Contamination levels in individual pistachios from each of the three pistachio categories considered

\begin{tabular}{|c|c|c|c|c|}
\hline \multicolumn{5}{|c|}{ contamination levels $(\mu \mathrm{g} / \mathrm{kg})$} \\
\hline \multicolumn{2}{|c|}{ early split sound kernel } & \multicolumn{2}{|c|}{ mouldy } & \multirow{2}{*}{$\begin{array}{c}\begin{array}{c}\text { growth split } \\
\text { sound kernel }\end{array} \\
7.88\end{array}$} \\
\hline 7 & 452 & 1.5 & 5333.44 & \\
\hline 14 & 971 & 2.68 & 5353.29 & 109.71 \\
\hline 22.88 & 2662 & 24.16 & 7370.58 & 203.55 \\
\hline 58.08 & 3013 & 27.8 & 8608.48 & 289.56 \\
\hline 72 & 3270 & 147.3 & 9256.8 & 2662.58 \\
\hline 109 & 5897 & 512.82 & 9559.76 & 5897.25 \\
\hline 175 & 8523 & 825.12 & 140828.8 & 8520.2 \\
\hline 203 & 26349 & 877.37 & 1418847 & \\
\hline 289 & 29363 & 942.92 & 1546770 & \\
\hline 318.08 & 34185 & 5237.82 & 2592381 & \\
\hline 445 & & & & \\
\hline
\end{tabular}

Table-2. Final calculation of contamination incidence: $P$ (contaminated $\cap$ category)

\begin{tabular}{cccc}
\hline & \multicolumn{3}{c}{ final contamination incidence } \\
\hline pistachio category & $\mathbf{1}^{\text {st }}$ class & $\mathbf{2}^{\text {nd }}$ class & $\mathbf{3}^{\text {rd }}$ class \\
\hline early split & $7.5456^{*} 10^{-5}$ & $1.50912^{*} 10^{-4}$ & $1.50912^{*} 10^{-4}$ \\
\hline cracks & $3.05666^{*} 10^{-5}$ & $6.11332^{*} 10^{-5}$ & $6.11332^{*} 10^{-5}$ \\
\hline mouldy & 0 & $8^{*} 10^{-6}$ & $1.69074^{*} 10^{-4}$ \\
\hline
\end{tabular}

Table-3. AFB1 contamination level distribution for $30 \mathrm{~kg}$ samples for each quality lot class

\begin{tabular}{|c|c|c|c|}
\hline \multirow{2}{*}{$\begin{array}{l}\text { AFB } 1 \text { contamination } \\
\text { level interval }(\mu \mathrm{g} / \mathrm{kg})\end{array}$} & \multicolumn{3}{|c|}{ proportion of samples } \\
\hline & $\begin{array}{c}\text { in } 1^{\text {st }} \begin{array}{l}\text { quality lot } \\
\text { class }\end{array} \\
\end{array}$ & $\begin{array}{c}\text { in } 2^{\text {nd }} \text { quality lot } \\
\text { class }\end{array}$ & $\begin{array}{c}\text { in } 3^{\text {rd }} \text { quality lot } \\
\text { class }\end{array}$ \\
\hline $\mathrm{O}-2$ & $96.1 \%$ & $74.5 \%$ & $5 \%$ \\
\hline $2.01-5$ & $3.2 \%$ & $12.8 \%$ & $12.8 \%$ \\
\hline $5.01-20$ & $0.7 \%$ & $9.6 \%$ & $36 \%$ \\
\hline $20.01-50$ & $\mathrm{O} \%$ & $2.1 \%$ & $22.7 \%$ \\
\hline $50.01-500$ & $\mathrm{O} \%$ & $0.8 \%$ & $22.1 \%$ \\
\hline$>500$ & $0 \%$ & $0 \%$ & $1.1 \%$ \\
\hline
\end{tabular}


Figure-1. AFB 1 contamination $(\mu \mathrm{g} / \mathrm{kg})$ PDF for $30 \mathrm{~kg}$ samples from $1^{\text {st }}$ quality lot class

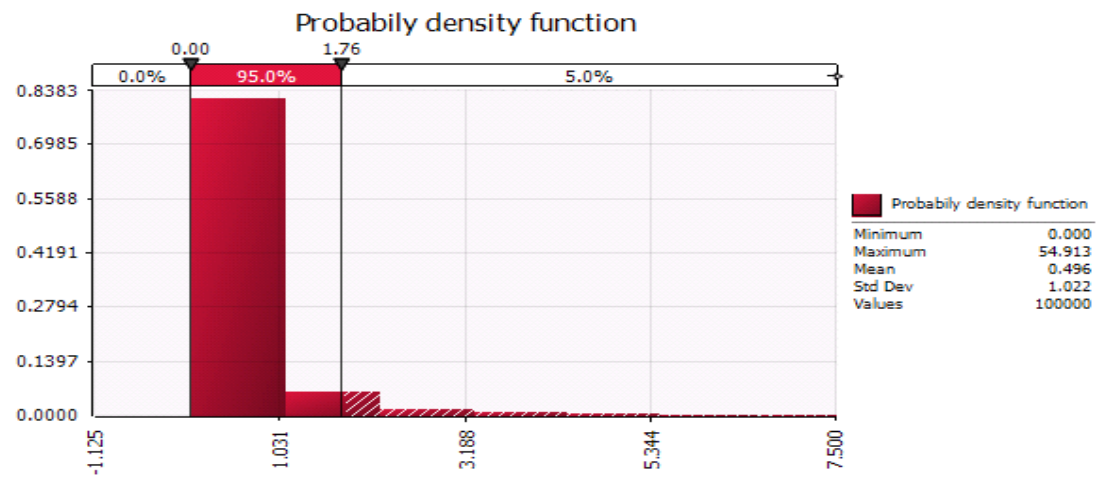

Figure-2. AFB1 contamination $(\mu \mathrm{g} / \mathrm{kg})$ PDF for $30 \mathrm{~kg}$ samples from $2^{\text {nd }}$ quality lot class

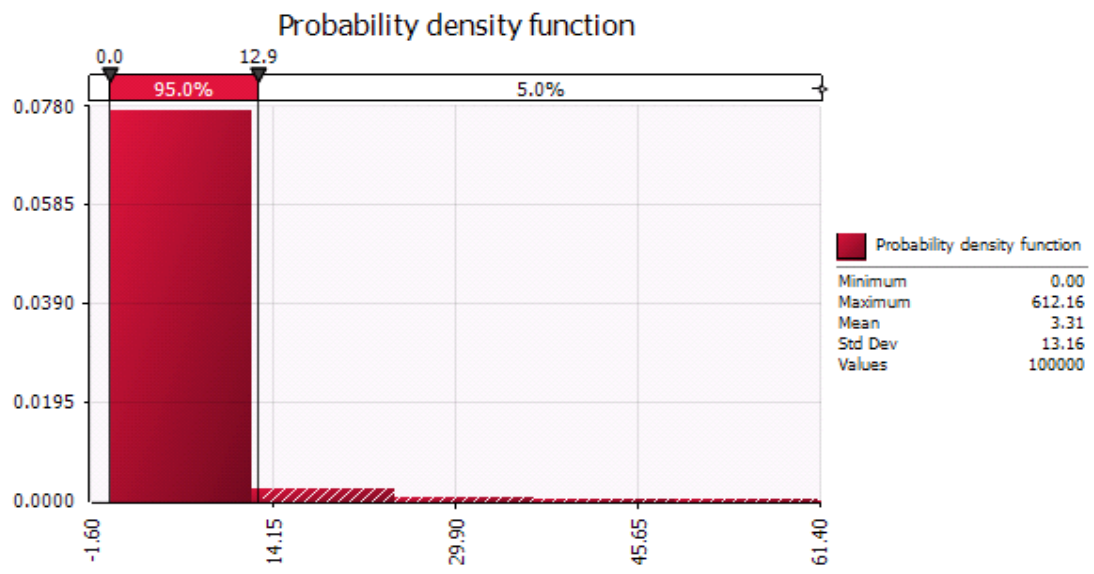

Figure-3. AFB 1 contamination $(\mu \mathrm{g} / \mathrm{kg})$ PDF for $30 \mathrm{~kg}$ samples from $3^{\text {rd }}$ quality lot class

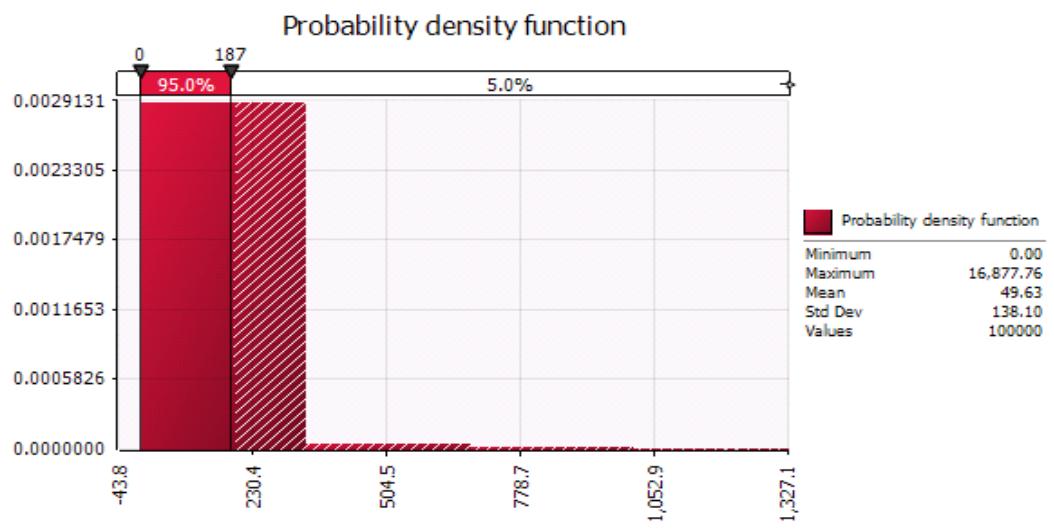




\section{APPENDIX}

Web Figure-1. Cumulative distribution function for the early split sound kernel pistachio category with aflatoxin concentration in $\mu \mathrm{g} / \mathrm{kg}$

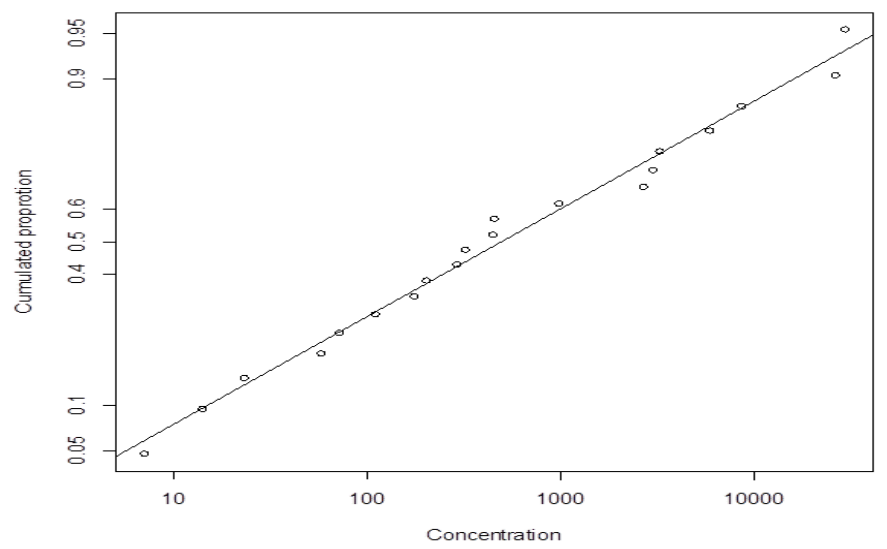

Web Figure-2. Cumulative distribution function for the cracked sound kernel pistachio category with aflatoxin concentration in $\mu \mathrm{g} / \mathrm{kg}$

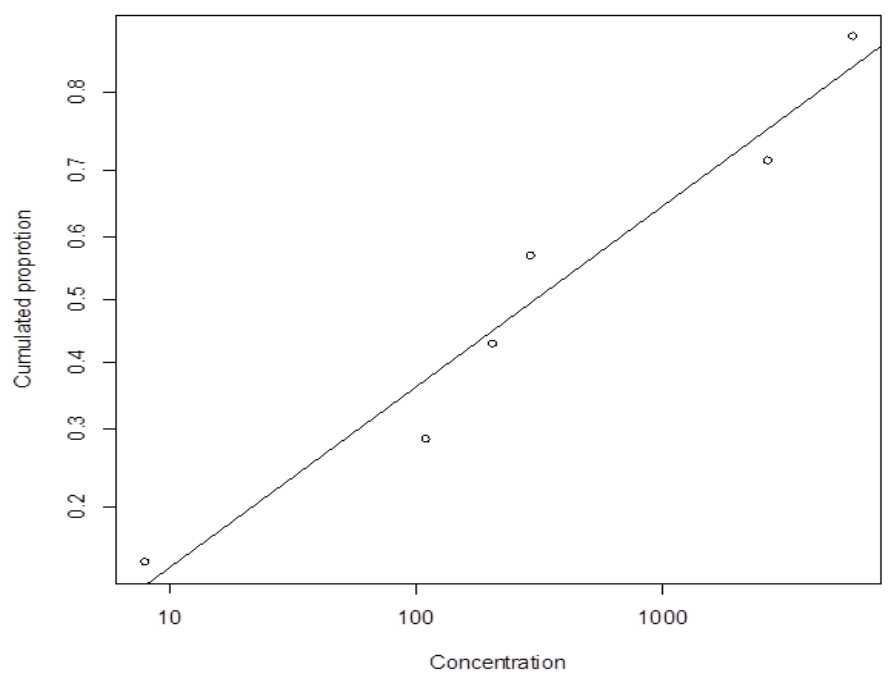

Web Figure-3. Cumulative distribution function for the mouldy pistachio category with aflatoxin concentration in $\mu \mathrm{g} / \mathrm{kg}$ 


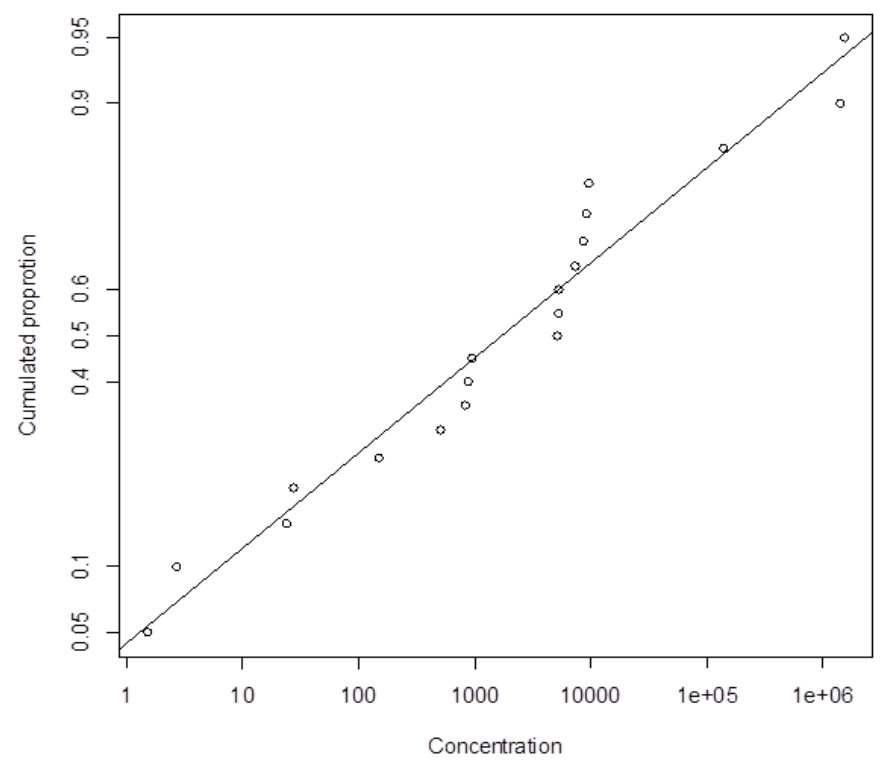

Web Figure-4. Visualization of the 3 quality lot classes' impact on contamination level

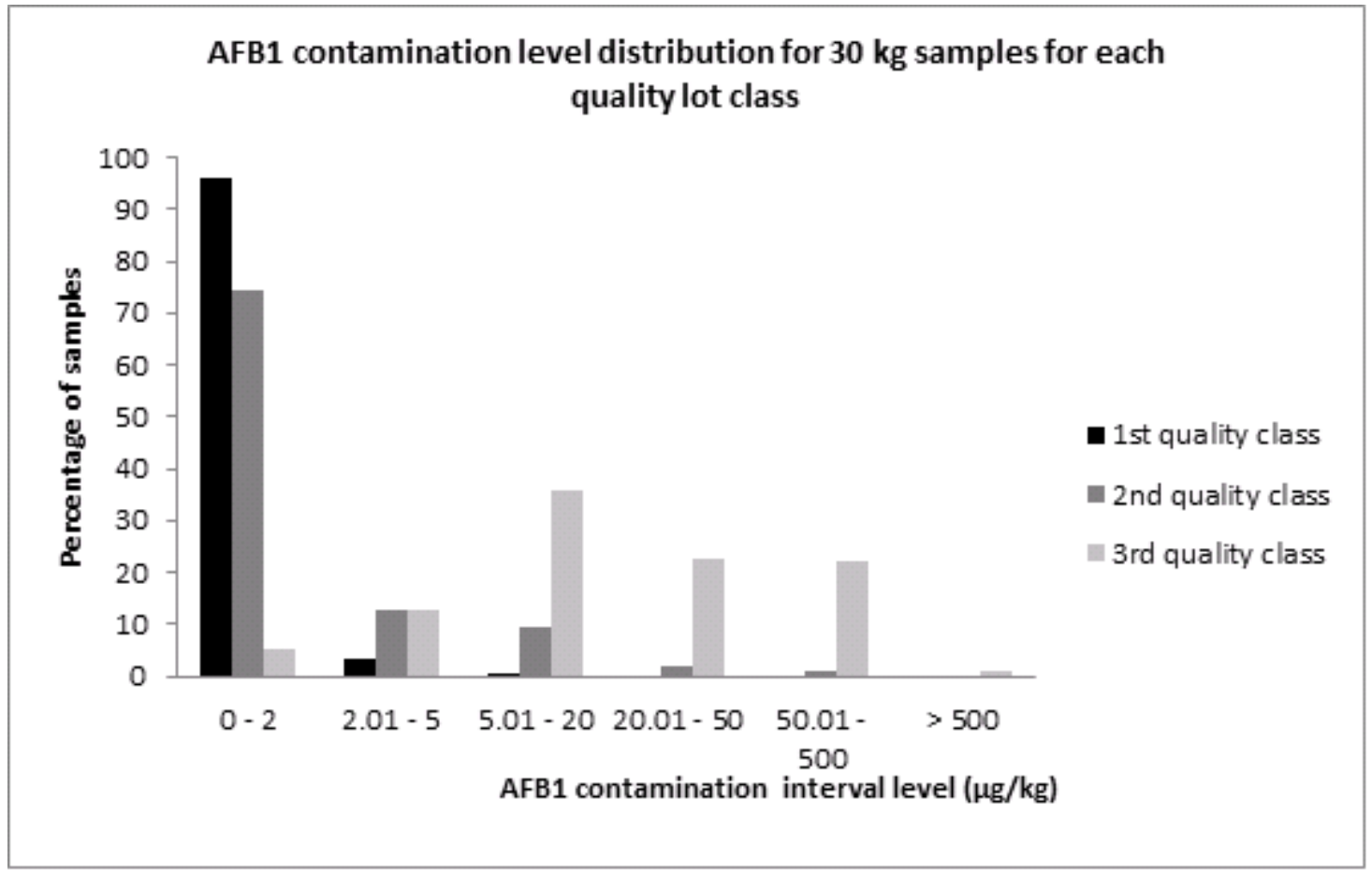


Journal of Food Technology Research, 2014, 1(1): 1-20

Web Figure-5. Visual validation of final simulation results distribution

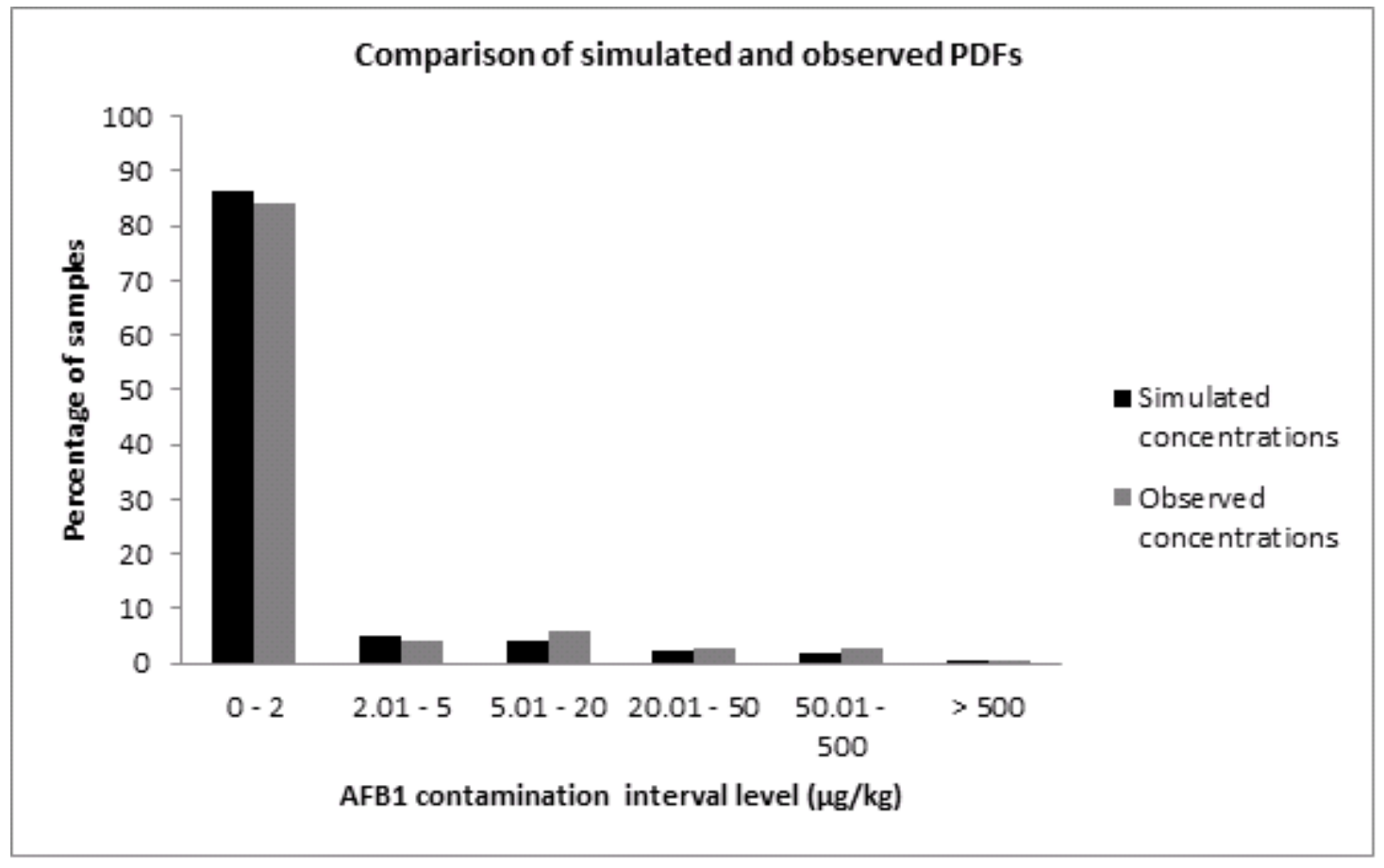


Web Figure-6. Impact of various probability levels tested for the two input binomial variables on the output distribution

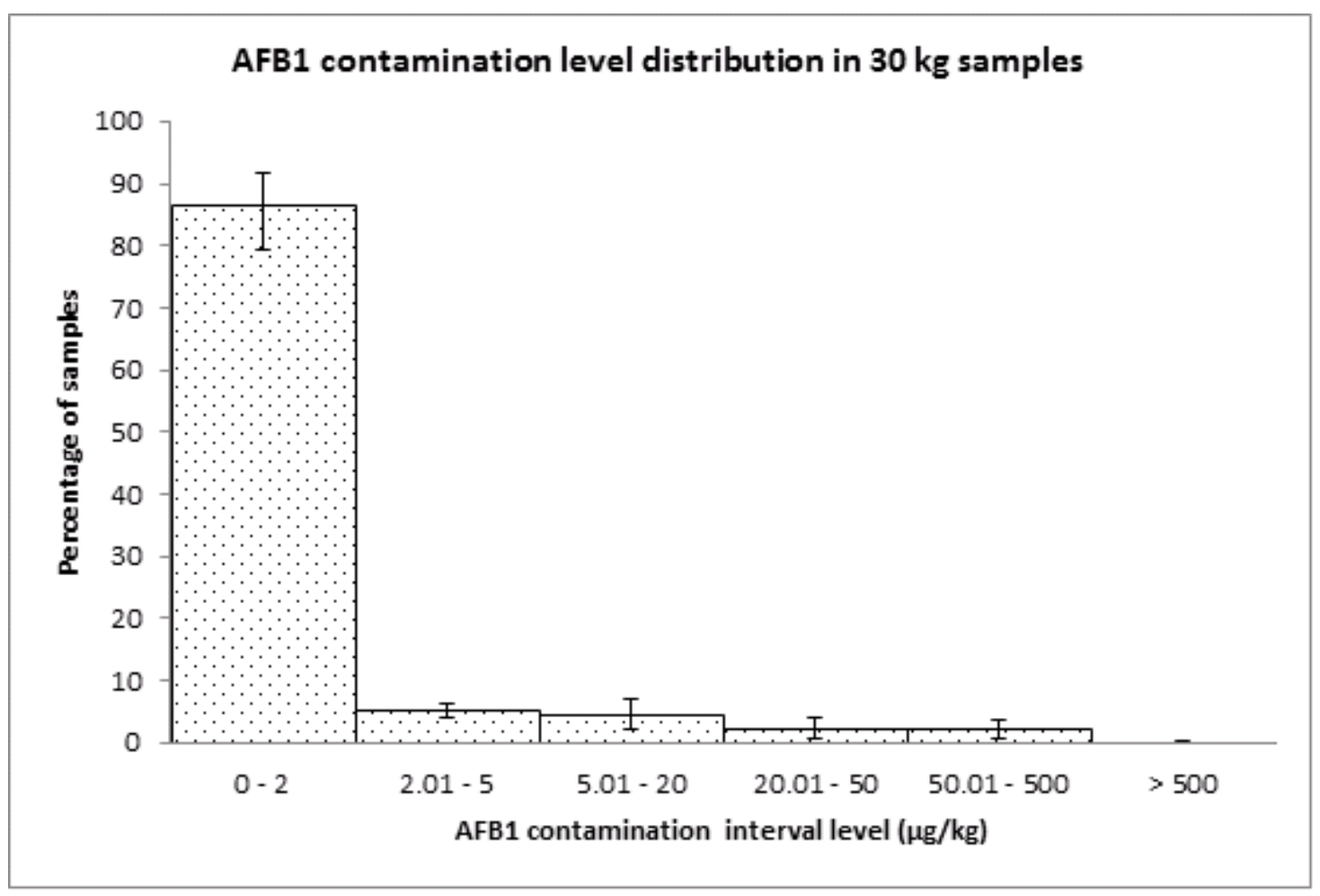

